\title{
Ketoconazole in the Treatment of Blepharitis
}

\author{
M. E. NELSON, G. MIDGLEY, N. R. BLATCHFORD \\ Sheffield, London and Wantage.
}

\begin{abstract}
Summary
A double masked, placebo controlled clinical trial, of topical $2 \%$ ketoconazole cream with lid hygiene, for the treatment of seborrhoeic and mixed seborrhoeic/staphylococcal blepharitis was conducted.

Forty patients with symptomatic blepharitis, 20 randomly allocated to ketoconazole, 20 to placebo were entered.

Treatment efficacy was assessed by improvement of symptoms using visual analogue scales, appearance of the eyelids and reduction in numbers of pityrosporum yeasts on the eyelids.

Both groups showed a similar reduction in symptoms, and signs of inflammation. The overall clinical impression in the ketoconazole group was better at week five than in the placebo group. Sixty nine per cent were either normal or markedly improved, as compared to $42 \%$ in the placebo group, although this was not statistically significant $(p<0.1$, one-sided Mann-Whitney). Pityrosporum numbers were reduced significantly in both groups during the treatment period.

Ketocanazole was no better than placebo at improving the symptoms of blepharitis. More ketoconazole treated patients had normal or markedly improved lids after treatment than the placebo group. Pityrosporum yeasts may play a role in blepharitis, and treatment with an antifungal has some advantages over conventional therapy.
\end{abstract}

Blepharitis is a common condition, which is difficult to treat. ${ }^{1}$ Patients complain of burning and itching of the eyes, photophobia, foreign body sensation, tearing and an ocular discharge that builds up on the lid margin to produce unsightly crusting.

The management of blepharitis is often inadequate, and patients frequently endure prolonged periods of ocular discomfort. In the long term more severe problems may develop, such as conjunctival scarring and drying, trichiasis and corneal ulcers and vascularisation.
Although our understanding of blepharitis has been improved with the introduction of a new classification of the disease, ${ }^{2}$ its pathogenesis particularly the role of bacteria and other organisms, is still unclear.

Staphylococcal blepharitis is often associated with the presence of coagulase positive Staphylococcus aureus on the eyelids. ${ }^{3,4}$ However, a large number of patients have coagulase negative Staphylococci, usually considered as non-pathogenic. ${ }^{5}$

Seborrhoeic blepharitis, a particularly chronic form of the disease, is associated with

From Department of Ophthalmology, Royal Hallamshire Hospital, Glossop Road, Sheffield. Department of Skin Infection, Institute of Dermatology, St John's Hospital for Diseases of the Skin, Lisle Street, London, and Research Programme Manager, Janssen Pharmaceutical Ltd., Grove, Wantage, Oxon.

Correspondence to: Mr. M. E. Nelson, Department of Opnthalmology, Royal Hallamshire Hospital, Glossop Road, Sheffield. 
generalised seborrhoeic dermatitis. Recent evidence points to the role of the yeast Pityrosporum (Malasszia) species, in seborrhoea and dandruff, for marked improvement in the condition has been obtained with the use of the antifungal agent, ketoconazole. ${ }^{6}$ This drug, a Pityrosporicidal agent with no antibacterial activity, ${ }^{7}$ is now used topically in the treatment of seborrhoeic dermatitis. ${ }^{8,9}$

Pityrosporum orbiculare and ovale have been identified on the lid margin from lid scrapings, in patients with blepharitis, in several studies, but the presence of the organism has been considered to be coincidental rather than causal in the disease. ${ }^{10-12}$

The standard treatment of blepharitis consists of meticulous lid hygiene, with cleansing and debridement of squames and crusts on the eyelids, with cotton buds and shampoo, followed by antibiotics or antiseptic solutions. ${ }^{13,14}$ Often this treatment is inadequate to control the problem and patients are rarely asymptomatic.

To record any therapeutic benefits of a Pityrosporicidal agent in the disease and consequently explore the possibility of a role in blepharitis for Pityrosporum, a double masked, placebo controlled trial of ketoconazole $2 \%$ cream as an adjunct to standard lid hygiene was conducted. The method and results of this trial are presented, together with a discussion of findings.

The trial was conducted in the Ophthalmology Department of the Royal Hallamshire Hospital, Sheffield, with full ethical committee approval.

\section{Method}

Patients were recruited for the study from the ophthalmic out-patient and casualty departments of the Royal Hallamshire Hospital. Forty symptomatic patients with seborrhoeic or mixed seborrhoeic/staphylococcal blepharitis not currently receiving treatment were assessed for inclusion in the trial.

Patients were excluded if they were receiving topical or systemic antibiotics or antiinflammatory drugs, or if significant active corneal disease was present. Contact lens wearers, women of child bearing potential not using contraception and patients known to be allergic to imidazole antifungals were also excluded.
Informed, written consent was obtained from all patients after a full explanation of the nature of the trial, before entry.

At first visit (week 1) details of the patients past ocular and medical history were taken, with particular attention to skin or rheumatic diseases. A detailed ocular examination was made, noting abnormalities of the lid position, eyelashes, meibomian glands, conjunctiva and cornea. Lid scaling, ulceration, meibomian gland inflammation and seborrhoea, conjunctival scarring and injection together with corneal punctate fluorescein staining were clinically graded as absent, mild, moderate and severe.

Swabs for bacterial culture were taken from the lid margins using standard techniques, with moistened swabs, placed in culture medium.

Skin scrapings from the eyelids were taken with a sterile surgical blade and examined after mounting in Parkers stain. ${ }^{15}$ Scrapings were also cultured on Dixon $\operatorname{agar}^{16}$ and incubated at $35^{\circ} \mathrm{C}$ for seven days. ${ }^{16}$

In addition more than half of the patients had a quantitative examination of lid mycology by the application of a strip of transparent adhesive tape to the upper lid which after removal contained an imprint of the superficial skin squames and fungi. After staining with Parker's stain the number of Pityrosporum yeasts on the eyelids could be counted and expressed as a number per square centimetre.

Each patient's symptoms were assessed using a visual analogue scale. They were asked to indicate the severity of five common symptoms encountered with blepharitis (itching, burning, discharge, foreign body sensation and lid scaling) by placing a cross on a line scale $10 \mathrm{~cm}$ long, from no symptoms to worst ever. Scoring of symptom severity was measured directly in millimetres with a ruler.

After examination patients were instructed in lid hygiene, using cotton buds moistened with Johnson and Johnson baby shampoo. Following lid hygiene they were asked to apply the cream provided on to the lid margins and the skin of the upper and lower lids. The cream either contained ketoconazole $2 \%$ in a lanolin base or placebo of identical formulation but without the antifungal agent. 
Patients were randomly allocated placebo or ketoconazole in a double masked manner.

Visual analogue scales were completed at weekly intervals for the nine weeks of the trial. Lid hygiene was continued for the whole study period but the cream was maintained only up to week five.

The patients were reassessed at two weekly intervals (weeks 1, 3, 5, 7 and 8) and the severity of the disease was recorded. Microbiology of the lids was repeated at each visit.

\section{Results}

Forty patients entered the trial; 20 used ketoconazole cream and 20 placebo cream. All patients used lid hygiene. Only three patients were withdrawn during the trial. One patient, on ketoconazole, developed a probable, but unconfirmed, hypersensitivity skin reaction one week after commencing the drug. She stopped the treatment, received topical hydrocortisone from her own general practitioner and suffered no long-term problems. A second patient receiving ketoconazole developed an unrelated viral keratoconjunctivitis and was withdrawn for treatment of this condition. The third patient was using placebo and developed marginal keratitis. This was treated by his general practitioner with topical steroid and he was therefore withdrawn from the trial.
In the ketoconazole group there were eleven females, and nine males. Mean age was 52 years with a range of 20 to 80 years. Mean duration of symptoms was two years.

The placebo group was made up of ten females and ten males, with a mean age of 49 years and an age range of $21-78$ years. Mean duration of symptoms was 3.5 years.

Where appropriate, statistical analysis with a hypothesis test for means, is given, unless otherwise stated.

\section{Visual Analogue Scales}

Visual analogue scales were completed weekly during the nine weeks of the trial. Scores of 0-100 were calculated by direct measurement along the scale and mean scores for each symptom (itching, burning, discharge, foreign body sensation and scaling) for weeks 1 to 9 are shown in Tables I and II.

The number of scales available for analysis varied each week because patients were withdrawn from the trial, occasionally could not attend or simply forgot to complete the scale at home. However the data available for each week was similar for both ketoconazole and placebo groups.

All patients improved with treatment, with a few differences between the ketoconazole and placebo groups. There was a wide varia-

Table I Results of visual analogue scales of symptom assessment in the ketoconazole treated group. SD represents 1 standard deviation from the mean

\begin{tabular}{|c|c|c|c|c|c|c|c|c|c|}
\hline & \multicolumn{9}{|c|}{ Week } \\
\hline & 1 & 2 & 3 & 4 & 5 & 6 & 7 & 8 & 9 \\
\hline \multicolumn{10}{|l|}{ Itching } \\
\hline Mean & 21 & 20 & 14 & 14 & 16 & 22 & 15 & 16 & 16 \\
\hline SD & 24 & 25 & 26 & 24 & 25 & 27 & 23 & 25 & 28 \\
\hline \multicolumn{10}{|l|}{ Burning } \\
\hline Mean & 22 & 30 & 20 & 21 & 21 & 19 & 15 & 16 & 13 \\
\hline SD & 26 & 31 & 27 & 25 & 30 & 26 & 25 & 25 & 19 \\
\hline \multicolumn{10}{|c|}{ Discharge } \\
\hline Mean & 24 & 17 & 15 & 17 & 13 & 16 & 10 & 13 & 9 \\
\hline $\mathrm{SD}$ & 31 & 23 & 25 & 24 & 23 & 26 & 19 & 24 & 25 \\
\hline \multicolumn{10}{|c|}{ Foreign body sensation } \\
\hline Mean & 24 & 26 & 24 & 25 & 23 & 26 & 19 & 12 & 16 \\
\hline SD & 27 & 26 & 26 & 28 & 28 & 26 & 27 & 22 & 24 \\
\hline \multicolumn{10}{|l|}{ Scaling } \\
\hline Mean & 21 & 16 & 14 & 17 & 16 & 10 & 11 & 14 & 19 \\
\hline $\mathrm{SD}$ & 24 & 23 & 26 & 23 & 25 & 19 & 20 & 25 & 33 \\
\hline $\mathrm{n}$ & 20 & 14 & 16 & 15 & 16 & 12 & 16 & 11 & 13 \\
\hline
\end{tabular}


Table II Results of visual analogue scales of symptom assessment in the placebo treated group. SD represents 1 standard deviation from the mean

\begin{tabular}{|c|c|c|c|c|c|c|c|c|c|}
\hline & \multicolumn{9}{|c|}{ Week } \\
\hline & 1 & 2 & 3 & 4 & 5 & 6 & 7 & 8 & 9 \\
\hline \multicolumn{10}{|l|}{ Itching } \\
\hline Mean & 30 & 25 & 19 & 28 & 22 & 17 & 17 & 19 & 20 \\
\hline $\mathrm{SD}$ & 33 & 20 & 25 & 26 & 23 & 12 & 23 & 26 & 21 \\
\hline \multicolumn{10}{|l|}{ Burning } \\
\hline Mean & 28 & 20 & 13 & 16 & 21 & 17 & 13 & 16 & 16 \\
\hline $\mathrm{SD}$ & 34 & 26 & 18 & 19 & 24 & 20 & 16 & 23 & 18 \\
\hline \multicolumn{10}{|c|}{ Discharge } \\
\hline Mean & 19 & 13 & 17 & 15 & 13 & 14 & 14 & 12 & 13 \\
\hline SD & 25 & 20 & 28 & 23 & 23 & 20 & 19 & 15 & 18 \\
\hline \multicolumn{10}{|c|}{ Foreign body sensation } \\
\hline Mean & 39 & 28 & 25 & 25 & 29 & 24 & 17 & 24 & 22 \\
\hline SD & 28 & 28 & 29 & 27 & 25 & 18 & 19 & 27 & 22 \\
\hline \multicolumn{10}{|l|}{ Scaling } \\
\hline Mean & 18 & 18 & 15 & 13 & 14 & 10 & 13 & 11 & 16 \\
\hline SD & 25 & 21 & 20 & 22 & 23 & 17 & 17 & 15 & 22 \\
\hline $\mathrm{n}$ & 20 & 17 & 15 & 13 & 14 & 10 & 13 & 11 & 16 \\
\hline
\end{tabular}

tion in scoring symptom severity between each patient, and this is reflected in the large standard deviations.

Foreign body sensation remained high until week seven suggesting that the lanolin base for both placebo and ketoconazole may have seen slightly irritant.

\section{Mycology}

Pityrosporum yeasts were identified in scrapings from 39 of the 40 patients in the study.

The mean Pityrosporum count from examination of sellotape impressions (expressed as a number of yeasts per $\mathrm{cm}^{2}$ ) for each group at each visit was calculated by taking the mean value from both eyes of each patient and then deriving the mean value from all of these values. The results are presented in Table III.

The mean number of Pityrosporum yeasts at week one was greater in the placebo group, than in the ketoconazole group. (difference $\left.1040 \mathrm{~cm}^{-2} \mathrm{p}=0.24\right)$.

Both groups showed a significant reduction in the number of organisms by week five (reduction of $1519 \mathrm{~cm}^{-2}$ in the ketoconazole group, and $2486 \mathrm{~cm}^{-2}$ in the placebo group $\mathrm{p}=0.01)$.

After the cream was stopped there was a

Table III Pityrosporum counts from sellotape impressions of eyelids in ketoconazole and placebo treated groups. Means are derived from pooled counts from both right and left lower eyelids. SD represents 1 standard deviation from the mean

\begin{tabular}{rrrrr}
\multicolumn{5}{c}{$\begin{array}{c}\text { Mycology } \\
\text { Week }\end{array}$} \\
\hline 1 & 3 & 5 & 7 & \multicolumn{1}{c}{9} \\
\hline & & 571 & 1550 \\
1386 & 663 & 547 & 571 & 2151 \\
13 & 332 & 631 & 393 & 7 \\
& 10 & 10 & 9 & 1285 \\
3106 & 1170 & 620 & 1013 & 706 \\
5195 & 2123 & 459 & 1209 & 7 \\
13 & 13 & 10 & 0 &
\end{tabular}


Table IV Mean clinical scores in both ketoconazole and placebo treated groups. SD represents 1 standard deviation from the mean

\begin{tabular}{|c|c|c|c|c|c|}
\hline & \multicolumn{5}{|c|}{$\begin{array}{c}\text { Clinical scores } \\
\text { Week }\end{array}$} \\
\hline & 1 & 3 & 5 & 7 & 9 \\
\hline \multicolumn{6}{|c|}{ Ketoconazole } \\
\hline Mean & 3.9 & 2.0 & 2.1 & 1.7 & 1.7 \\
\hline $\mathrm{SD}$ & 0.9 & 0.7 & 1.3 & 0.8 & 1.1 \\
\hline Number & 20 & 16 & 16 & 14 & 14 \\
\hline \multicolumn{6}{|l|}{ Placebo } \\
\hline Mean & 4.4 & 2.2 & 1.5 & 2.0 & 1.7 \\
\hline $\mathrm{SD}$ & 1.3 & 1.5 & 0.9 & 1.4 & 1.2 \\
\hline Number & 20 & 18 & 19 & 12 & 14 \\
\hline
\end{tabular}

gradual rise in the yeast count. At week seven the Pityrosporum count in the placebo treated group was higher than the ketoconazole group. (difference $442 \mathrm{~cm}^{-2} \mathrm{p}=.15$ ).

Only 29 positive cultures (from 324 scrapings) were obtained. Pityrosporum ovale was grown in 23, and $P$ orbiculare in six.

\section{Clinical Features}

Lid scaling, conjunctival injection and seborrhoea were judged as absent, mild, moderate or severe, and scored from 0-3. The mean total score for both ketoconazole and placebo groups are shown in Table IV.

Both groups showed a significant improvement in mean score from weeks one to three, which was sustained $(\mathrm{p}<0.1$, one-sided Mann-Whitney). There was no difference between the two groups.

At week five, the overall clinical impression of the severity of blepharitis was assessed by the author, with the patients' treatment still masked. The eyes were grouped as normal, markedly improved, moderately improved, unchanged or deteriorated.

No patient had deteriorated whilst in the trial.

Of those patients receiving ketoconazole, $69 \%(11 / 16)$ were either normal or markedly improved, as compared to only $42 \%(8 / 19)$ receiving placebo, although this did not reach statistical significance $(0.1>p>0.05$, onesided Mann-Whitney).

\section{Bacteriology}

The results form the bacterial swabs are presented in Tables V and VI.

The most commonly cultured organisms were coagulase-negative Staphylococci. There was no difference between the ketoconazole and the placebo groups, in bacterial colonisation of the lids. Both groups showed a

Table $\mathbf{V}$ Bacterial cultures from eyelids in ketoconazole. Numbers represent number of patients with bacterial growth (percentage)

\begin{tabular}{lccccc}
\hline \multicolumn{5}{c}{$\begin{array}{c}\text { Bacteriology } \\
\text { Week }\end{array}$} \\
\cline { 2 - 5 } & 1 & 3 & 5 & 7 & 9 \\
\hline Ketoconazole & & & & \\
Coagulase negative & $17(85)$ & $11(64)$ & $7(41)$ & $10(83)$ & $10(90)$ \\
$\quad$ Staphylococci & $3(15)$ & $4(23)$ & $4(23)$ & $2(16)$ & 0 \\
Diphtheroids & 0 & 0 & $2(11)$ & $2(16)$ & $1(9)$ \\
Staphylococci aureus & $1(5)$ & 0 & $1(5)$ & 0 & 0 \\
Coliforms & $1(5)$ & $2(11)$ & 0 & 0 & $1(9)$ \\
Streptococci & $2(10)$ & $5(29)$ & $6(35)$ & 1 & 0 \\
Not-specified & 0 & 0 & $1(5)$ & 0 & 0 \\
No growth & 20 & 17 & 17 & 12 & 11 \\
$\quad$ N & & & & & 0 \\
\hline
\end{tabular}


Table VI Bacterial cultures from eyelids in Placebo group. Numbers represent number of patients with bacterial growth (percentage)

\begin{tabular}{|c|c|c|c|c|c|}
\hline & \multicolumn{5}{|c|}{$\begin{array}{c}\text { Bacteriology } \\
\text { Week }\end{array}$} \\
\hline & 1 & 3 & 5 & 7 & 9 \\
\hline \multicolumn{6}{|l|}{ Placebo } \\
\hline \multicolumn{6}{|l|}{ Coagulase negative } \\
\hline Staphylococci & $18(90)$ & $15(78)$ & $12(66)$ & $12(80)$ & $6(66)$ \\
\hline Diphtheroids & $6(30)$ & $1(5)$ & $3(16)$ & $3(20)$ & $1(11)$ \\
\hline Staphylococci aureus & $1(5)$ & $1(5)$ & $1(5)$ & $1(6)$ & 0 \\
\hline Coliforms & $1(5)$ & 0 & 0 & 0 & 0 \\
\hline Streptococci & 0 & $1(5)$ & 0 & 0 & 0 \\
\hline Not-specified & $1(5)$ & $2(10)$ & $6(33)$ & $2(11)$ & $3(33)$ \\
\hline No growth & 0 & $2(10)$ & 0 & 0 & 0 \\
\hline $\mathbf{N}$ & 20 & 19 & 18 & 15 & 9 \\
\hline
\end{tabular}

slight reduction in coagulase negative staphylococcal cultures, during the trial but few had no bacterial growth. The total number of bacterial species cultured exceeds the total cultures undertaken as some patients had multiple bacterial growths.

\section{Discussion}

Blepharitis is a chronic disease with inflammation of the eyelids leading to secondary conjunctivitis and ocular discomfort. It occurs commonly, frequently beginning in childhood, and continuing throughout life.

In many cases, the problems associated with blepharitis are minor, and at most irritating. However in a considerable number of patients it is a cosmetic problem, a source of constant ocular and peri-ocular discomfort, and a cause of styes, chalazia and trichiasis. In severe cases, it is associated with the development of corneal ulceration, vascularisation, thinning and even perforation.

Initially classified as squamous and ulcerative, by Thygeson in $1946,{ }^{10}$ a more comprehensive classification proposed by McCulley et $a .^{2}$ describe the following categories of blepharitis:

(1) Staphylococcal

(2) Seborrhoeic

(a) Alone

(b) Mixed seborrhoeic/staphylococcal

(c) Seborrhoeic with meibomian seborrhoea

(d) Seborrhoeic with secondary meibomitis

(3) Primary meibomitis

(4) Other, including atopic, psoriatic, fungal, etc.
Of these, the mixed seborrhoeic/staphylococcal variety is the most common, and makes up most of the patients included in this study.

Staphylococci, particularly $S$. aureus and coagulase negative $S$. epidermidis, have been implicated in the aetiology of blepharitis for many years, but their actual role in the pathogenesis of the disease is as yet unclear..$^{3-5,16}$ Theories include the production of staphylococcal toxin and lipases, direct bacterial pathogenicity, and tissue hypersensitivity to bacterial antigen. ${ }^{18,19}$

Patients with a Staphylococcal blepharitis have exacerbations and remissions of the disease, with periods of acute, short-lived inflammation. These attacks are usually accompanied by conjunctivitis and keratitis. The lids show hard brittle scales of desquamated skin, often forming around, and being lifted from the surface by the lashes, and known as collarettes. Beneath these collarettes are shallow ulcers. Secondary changes in the lids are telangiectasis, trichiasis, poliosis and madarosis.

Lid scales in seborrhoeic dermatitis are usually greasy, or oily, and local lid inflammation less severe. Staphylococcal superinfection is frequent, producing the common mixed picture. ${ }^{20}$

The meibomian glands are specialised sebaceous glands in the posterior lamella of the eyelid. They may be primarily or secondarily involved in blepharitis. Meibomian seborrhoea is characterised by the production of copious amounts of lipid that appears on the lid margin as small oil globules, or as col- 
lections of waxy material. Sometimes the secretion is in such large amounts that it accumulates as a frothy discharge at the lateral canthus. ${ }^{21}$

Meibomian gland inflammation may occur, with acute, or chronic meibomitis contributing to the disease morbidity. Examination of these glands in vivo and histologically, have shown gross abnormalities of the gland structure. $^{22}$

$P$. ovale and $P$. orbiculare have often been implicated in seborrhoeic dermatitis. Controversy has existed as to whether the organism is primary in the aetiology of seborrhoea or merely a co-incidental saprophyte. The two species are separated by the shape of the cell, oval in $P$. ovale and spherical in $P$. orbiculare, although taxonomists currently classify them as forming one species in the genus Malasse$z i a{ }^{23}$ They both require lipid for growth and therefore culture is not straightforward. Attempts were made to isolate the yeasts from eyelid specimens in this study but difficulty in obtaining sufficient scrapings and the problem with transportation to a laboratory sufficiently equipped for its culture resulted in a low recovery rate. From a total of 324 cultures, $P$. orbiculare was grown from six patients and $P$. ovale from 23 . By contrast the organism was seen in virtualy all.

Pityrosporum spp. in skin disease is reviewed by Shuster, ${ }^{24}$ and its role as the primary agent in pityriasis versicolor, seborrhoeic dermatitis and dandruff is now established.

Similarly these species have been implicated in blepharitis, and more especially in seborrhoeic blepharitis. Thygeson describes $P$. ovale in all of his patients with seborrhoeic blepharitis, having found it in lid scrapings. ${ }^{10}$ Gots et al, confirmed this in their series but were disappointed in not being able to transfer the disease to animals or human volunteers. ${ }^{11}$ However Parunovic in detailed animal inoculation experiments was unable to prove a causal relationship between Pityrosporum and the disease. He concluded that the yeast existed as a mere saprophyte on the lids of most patients, and was not pathogenic. ${ }^{12}$

This study has similarly confirmed the presence of Pitysrosporum often in large numbers on the lids of patients with blepharitis. It is clear from the results presented that the improvement in some symptoms and the severity of the disease parallels the reduction in the number of the organisms seen. However this does not prove that the yeast is producing the disease, as an improvement in the condition may make the environment hostile enough to decrease numbers.

The effectiveness of oral ketoconazole in the clearance of Pityrosporum folliculitis was reported in 1981 by Neumann and Berretty, ${ }^{25}$ and again in 1982 by Ford et al. ${ }^{26}$ This was followed by the success of therapy in seborrhoeic dermatitis, by Ford et al. in 1984, again with oral ketoconazole. ${ }^{6}$ Because of the risk of hepatotoxicity in long-term systemic treatment with ketoconazole, the effect of topical ketoconazole shampoo and cream, was tested in 1987 by Carr et al. ${ }^{8}$ and Green et al. ${ }^{9}$ With both preparations a favourable response was reported.

This study has evaluated the use of a fourweek course of topical ketoconazole cream in the management of blepharitis, by assessing any improvement in symptoms and signs of inflammation.

A reduction in yeast count was seen in both ketoconazole and placebo treated patients so that lid hygiene and possibly the mechanical effect of using a cream on the eyelid is sufficient to reduce Pityrosporum numbers on the lid margin. Johnson and Johnson Baby Shampoo contains propylene glycol which is effective in the treatment of pityriasis versicolor, and may in itself be pityrosporicidal. ${ }^{7}$ This of course provides no proof that the organism is a primary pathogen as it may well only reside in inflamed and oedematous skin, but in studies with seborrheoic dermatitis a reduction in the organism did also correlate with improvement in the condition. Further studies with antipityrosporal agents alone need to be conducted to establish if seborrhoeic blepharitis has a fungal aetiology.

There was a difference in the overall clinical appearance of the condition with $69 \%$ of patients in the ketoconazole group normalised or markedly improved, as compared to only $42 \%$ of the placebo group.

The clinical symptoms of the two groups, as recorded with the visual analogue scales, were 
more or less similar. However some important problems are worthy of mention in the interpretation of the visual analogue scales.

The initial scoring of the symptoms was often low, so that the mean score before treatment was around 30 . This made any significant improvement due to treatment very difficult to detect, purely on the scale alone. The low initial scores are partly due to a poor understanding of the nature of the scale by the patient with a consequent improvement in understanding as the weeks went by. Often scores were given as low when the patient expressed their symptoms as very severe. The protocol required the patients record their own score after an explanation without help from the investigator, so this error had to remain.

Furthermore, several patients stated that their symptoms at the time of filling in the scale were relatively mild although the previous day they had been more severe. Blepharitis is a variable condition and so a single assessment of symptom severity prior to treatment could be artificially low.

No attempt has been made to explain the pathogenesis of blepharitis and in particular the specific role of Pityrosporum spp. The role of this yeast in seborrhoeic dermatitis is clear, although the actual pathogenesis remains unknown.

Pityrosporum is lipophilic and contains enzymes for the breakdown of fatty acids and sterols. ${ }^{7}$ Lipoxygenase activity has also been demonstrated. ${ }^{27}$ Whether this is relevant in the development of symptoms is as yet unknown. It is possible that the yeast may not be primarily pathogenic but that a hypersensitivity response occurs in some people. Removal of the yeast will still, nonetheless lead to resolution of the condition by removing the antigenic stimulus. This mechanism is already accepted as the probable explanation of staphylococcal lid disease, especially when nonpathogenic staphylococci are involved. A rabbit model of blepharitis requires the prior sensitisation of the animal with intravenous antigen, before the introduction of the bacteria on the lid margin will produce the disease. ${ }^{28}$ It is possible that this mechanism may occur in Pityrosporal lid disease.
References

${ }^{1}$ Dougherty JM and McCulley JP: Comparative bacteriology of chronic blepharitis. Br J Ophthalmol 1984, 68: 524-8.

${ }^{2}$ McCulley JP, Dougherty JM, Deneau DG: Classification of chronic blepharitis. Ophthalmology 1982, 89: 1173-80.

${ }^{3}$ Galin MA: Bacteriological aspects of blepharitis. Arch Ophthalmol 1962, 67: 746-9.

${ }^{4}$ Smolin G and Okumoto M: Staphylococcal blepharitis. Arch Ophthalmol 1977, 95: 812-6.

${ }^{5}$ McCulley JP and Dougherty JM: Bacterial aspects of chronic blepharitis. Trans Ophthalmol Soc UK 1986, 105: 314-18.

${ }^{6}$ Ford GP, Farr PM, Ive FA, Shuster S: The response of seborrhoeic dermatitis to ketoconazole. $\mathrm{Br} J$ Dermatol 1984, 111: 603-7.

${ }^{7}$ Hay RJ and Midgley G: Pathogenic mechanisms of Piyrosporum infection. In Seborrhoeic dermatitis and dandruff-a fungal disease. International Congress and Symposium Series Number 132, Editors Shuster S and Blatchford N, Royal Society of Medicine Services, London and New York 1988, pp. 13-19.

${ }^{8}$ Carr MM, Pryce DM, Ive FA: Treatment of seborrhoeic dermatitis with ketoconazole I. $\mathrm{Br} J \mathrm{Der}$ matol 1987, 116: 213-6.

${ }^{9}$ Green CA, Farr PM, Shuster S: Treatment of seborrhoeic dermatitis with ketoconazole II. $\mathrm{Br} J \mathrm{Der}$ matol 1987, 116: 217-21.

${ }^{10}$ Thygeson P: Aetiology and treatment of blepharitis. Arch Ophthalmol 1956, 36: 445-77.

${ }^{11}$ Gots JS, Thygeson P, Waisman M: Observations on Pityrosorum ovale in seborrhoeic blepharitis and conjunctivitis. Am J Ophthalmol 1947, 30: 1485-95.

${ }^{12}$ Parunovic A and Halde C: Pityrosporum orbiculare; Its possible role in seborrhoeic blepharitis. Am J Ophthalmol 1967, 63: 815-20.

${ }^{13}$ Polack FM and Goodman DF: Experience with a new detergent lid scrub in the management of chronic blepharitis. Arch Ophthalmol 1988, 106: 719-20.

${ }^{14}$ Leibowitz HM and Capino D: Treatment of chronic blepharitis. Arch Ophthalmol 1988, 106: 720.

${ }^{15}$ Cohen MM: A simple procedure for staining tinea versicolor (M. furfur) with fountain pen ink. J Invest Dermatol 1954, 22: 9-10.

${ }^{16}$ Van Abbe NJ: The investigation of dandruff. $J$ Soc Cosmet Chem 1964, 15: 609-30.

${ }^{17}$ Seal DV: Bacterial classification and diagnosis. Trans Ophthalmol Soc UK 1986, 105: 32-6.

${ }^{18}$ Seal DV, McGill JI, Jacobs P, Liakos GM, Goulding $\mathrm{NJ}$ : Microbial and immunological investigations of chronic non-ulcerative blepharitis and meibomianitis. Br J Ophthalmol 1985, 69: 604-11.

${ }^{19}$ Dougherty JM and McCulley JP: Bacterial lipases and chronic blepharitis. J Invest Ophthalmol Vis Sci 1986, 27: 486-91.

${ }^{20}$ Keith CG: Seborrhoeic blepharo-kerato-conjunctivitis. Trans Ophthalmol Soc UK 1967, 87: 85-103.

${ }^{21}$ McCulley JP and Sciallis GF: Meibomian keratoconjunctivitis. Am J Ophthalmol 1977, 84. 788-93. 
${ }^{22}$ Gutgesell VJ, Stern GA, Hood CI: Histopathology of meibomian gland dysfunction. Am J Ophthalmol 1982, 94: 383-7.

${ }^{23}$ Midgley G: The diversity of Pityrosporum (Malassezia) yeasts in vivo and in vitro. Mycopathologica (1989 in press).

${ }^{24}$ Shuster S: The aetiology of dandruff and the mode of action of therapeutic agents. Br J Dermatol 1984, 111: $235-42$.

${ }^{25}$ Neumann HAM and Berretty PJM: Treatment of Pityrosporum folliculitis with ketoconazole.
Mykosen 1981, 24: 167-71.

${ }^{26}$ Ford GP, Ive FA, Midgley G: Pityrosporum folliculitis and ketoconazole. Br J Dermatol 1982, 107: 691-5.

${ }^{27}$ Nazzaro-Porro M, Passi S, Picardo M, Mercantini R, Breathnach AS: Lipoxygenase activity of Pityrosporum in vitro and in vivo. J Invest Dermatol 1986, 87: 108-12.

${ }^{28}$ Mondino BJ, Caster AI, Dethlefs B: A rabbit model of staphylococcal blepharitis. Arch Opthalmol 1987, 105: 409-12. 